\title{
CDC20 promotes the progression of hepatocellular carcinoma by regulating epithelial-mesenchymal transition
}

\author{
GANG YANG $^{1 *}$, GUAN WANG $^{2 *}$, YONGFU XIONG ${ }^{1,3^{*}}$, JI SUN $^{1}$, WEINAN LI ${ }^{1}$, TAO TANG ${ }^{1}$ and JINGDONG LI $^{1,3}$ \\ ${ }^{1}$ Department of Hepatocellular Surgery, ${ }^{2}$ Physical Examination Center, Affiliated Hospital of \\ North Sichuan Medical College, Nanchong, Sichuan $637007 ;{ }^{3}$ Institute of Hepato-Biliary-Intestinal Disease, \\ North Sichuan Medical College, Nanchong, Sichuan 637000, P.R. China
}

Received September 27, 2020; Accepted March 29, 2021

DOI: $10.3892 / \mathrm{mmr} .2021 .12122$

\begin{abstract}
Hepatocellular carcinoma (HCC) is a type of primary liver cancer, which is associated with high mortality. $\mathrm{HCC}$ is one of the most common malignant tumors worldwide. Cell division cycle 20 (CDC20) has been reported to be associated with the development of various malignant tumors and epithelial-mesenchymal transition (EMT) has been reported to be involved in the malignant metastasis of HCC. Therefore, the present study hypothesized that $\mathrm{CDC} 20$ may participate in the malignant biological behavior of HCC via EMT. The present study analyzed the expression levels of CDC20 in $\mathrm{HCC}$ and the association between $\mathrm{CDC} 20$ and poor prognosis. Furthermore, the effects of CDC20 on the proliferation, invasion and migration of HCC cells were examined using proliferation, migration and invasion assays. Finally, alterations in EMT were analyzed. The results revealed that CDC20 was highly expressed in HCC and HCC cell lines $(\mathrm{P}<0.05)$, and its high expression level was significantly associated with poor prognosis in patients with HCC $(\mathrm{P}<0.05)$. CDC20 silencing inhibited the proliferation, migration and invasion of HCC cells. Furthermore, CDC20 silencing increased the expression levels of E-cadherin, and decreased the expression levels of $\mathrm{N}$-cadherin, vimentin and $\mathrm{Ki}-67$. In conclusion, the present study reported that CDC20 may be a novel therapeutic target in HCC and CDC20 could promote the progression of $\mathrm{HCC}$ by regulating EMT.
\end{abstract}

Correspondence to: Professor Jingdong Li, Department of Hepatocellular Surgery, Affiliated Hospital of North Sichuan Medical College, 63 Wenhua Road, Nanchong, Sichuan 637007, P.R. China

E-mail: 1i-jingdong@hotmail.com

${ }^{*}$ Contributed equally

Key words: cell division cycle 20, epithelial-mesenchymal transition, hepatocellular carcinoma, proliferation, migration, invasion

\section{Introduction}

Hepatocellular carcinoma (HCC) is a common type of cancer that originates from hepatocytes and accounts for $70-90 \%$ of primary liver cancer cases worldwide (1). Approximately 500,000 cases of HCC are diagnosed globally every year, and HCC is the fifth most common type of cancer in men and the eighth most common in women (2). If HCC is not treated, patients succumb quickly, and the global 5-year survival rate is only $5 \%(3-5)$. Therefore, it is necessary to elucidate the specific mechanism underlying the occurrence and development of HCC, and to provide a scientific basis for the diagnosis and treatment of HCC.

Cell division cycle 20 (CDC20) is a cell cycle checkpoint regulator (6). CDC20 and another regulator E-cadherin can directly bind and activate the anaphase-promoting complex (APC), which has an important role in the process of cells entering and exiting mitosis (7). Recently, an increasing number of studies have shown that CDC20 is upregulated in various types of human malignant tumor, including pancreatic ductal adenocarcinoma, breast cancer, glioblastoma and gastric cancer; in addition, CDC20 may be closely associated with the poor prognosis of various types of cancer (8-11). A previous study reported that overexpression of CDC20 may promote the resistance of castration-resistant prostate cancer cell lines to docetaxel in a Bim-dependent manner (12). Zhang et al (13) reported that reducing the expression of CDC20 may inhibit the expression of $\mathrm{CD} 44^{+}$prostate cancer stem cells. Moreover, CDC20 has been shown to promote the degradation of AXIN1, reduce the phosphorylation of $\beta$-catenin and promote the nuclear translocation of $\beta$-catenin, thus enhancing the self-renewal ability of $\mathrm{CD} 44^{+}$prostate stem cells (13). However, to the best of our knowledge, the association of CDC20 with HCC has only been determined through bioinformatics analysis, and no research has been conducted on the association of CDC20 with cell function and related molecular signaling pathways in HCC (13). Therefore, the present study aimed to assess the specific mechanism underlying the effects of CDC20 on HCC.

Epithelial-mesenchymal transition (EMT) refers to the process by which cells transition from an epithelial phenotype to a mesenchymal phenotype under specific physiological or pathological conditions. Notably, EMT has been confirmed to 
play an important role in HCC (14). The process of EMT causes loss of expression of E-cadherin, claudin, occludin and other connecting molecules in epithelial cells, destroys cell polarity and promotes some lytic enzymes that are involved in degradation of the extracellular matrix and basement membrane (15). Increased expression of matrix metalloproteinases, which occurs during EMT, destroys the histological barrier of tumor cell invasion, facilitating the separation of tumor cells from the primary tumor, invasion and metastasis (16). These findings indicated that EMT may serve an important role in promoting HCC metastasis.

The present study aimed to investigate the effect of CDC20 on the poor prognosis of patients with HCC and the biological functions of HCC cells. In addition, the molecular mechanism by which CDC20 affects malignant progression of HCC through EMT was investigated, in order to provide potential strategies for the prognosis and treatment of patients with HCC.

\section{Materials and methods}

Patients and tumor specimens. A total of $71 \mathrm{HCC}$ and paired adjacent tissue specimens were collected from Department of Hepatocellular Surgery, Affiliated Hospital of North Sichuan Medical College (Nanchong, China) between January 2010 and May 2013. The mean age of the patients was 61 years (age range, 30-77 years), and the cohort consisted of 30 men and 41 women (Table I). Patients with HCC were followed up for 90 months after surgery, in order to evaluate survival rate (patients were followed up by phone every 4 months, and the total follow-up period was 90 months). All procedures involving human participants were approved by the Ethics Committee of Affiliated Hospital of North Sichuan Medical College and were conducted in accordance with the Declaration of Helsinki. The corresponding clinicopathological data were obtained from Affiliated Hospital of North Sichuan Medical College. All patients who participated in the experiment provided written informed consent.

The detailed inclusion criteria were as follows: i) HCC was confirmed by postoperative pathology, and the histopathological diagnosis was clear; ii) patients were diagnosed with HCC for the first time without distant metastasis; iii) patients had not received any treatment prior to surgery; iv) no other serious malignant disease had been diagnosed; v) the clinical, pathological and surgical data were complete; vi) the follow-up information was complete and available.

Immunohistochemical staining. A total of $71 \mathrm{HCC}$ tissues were fixed in $10 \%$ formalin for $12 \mathrm{~h}$ at room temperature, embedded in paraffin and cut into $4-\mu \mathrm{m}$ sections. The tissue sections were then used to generate tissue microarray cores (1.5-mm diameter). The microarrays were deparaffinized in xylene I for $15 \mathrm{~min}$ and xylene II for $15 \mathrm{~min}$ at room temperature, and were rehydrated in a graded ethanol series $(100,95$, 80 and $75 \%$ ethanol, 5 min each). Subsequently, the microarrays were incubated with $3 \% \mathrm{H}_{2} \mathrm{O}_{2}$ for $30 \mathrm{~min}$ at $37^{\circ} \mathrm{C}$ and with $5 \%$ goat serum (Origene Technologies, Inc.) for $15 \mathrm{~min}$ at $37^{\circ} \mathrm{C}$ to block non-specific binding. The microarrays were then incubated with a monoclonal anti-CDC20 antibody $(1: 2,000$; cat. no. ab215908; Abcam) at $4^{\circ} \mathrm{C}$ overnight. Subsequently, the sections were incubated with a secondary biotin-labeled IgG antibody (1:100; cat. no. SAP-9100; Origene Technologies, Inc.) at $37^{\circ} \mathrm{C}$ for $30 \mathrm{~min}$. The visualization signal was detected using 3,3'-diaminobenzidine (Boster Biological Technology) at room temperature for $10 \mathrm{sec}$. Slides were visualized using a light microscope (magnification, x100; Zeiss AG).

The immunostaining results were analyzed using the following scoring parameters: Staining intensity [according to the color development degree of the positive marker (range, 0-3): No staining, negative (score, 0); light yellow staining, weak (score, 1); brown-yellow staining, moderate (score, 2); and brown black staining, strong (score, 3)] and percentage of positive cells (range, $0-4: 0,<5 \% ; 1,6-25 \% ; 2,26-50 \%$; $3,51-75 \%$; and $4,76-100 \%$ ). Total score was determined by adding the staining intensity score to the percentage of positive cells score. Slides with a total score $<4$ were defined as low CDC20 expression, whereas slides with a score $\geq 4$ were defined as high CDC20 expression (17).

Cell lines and transfection. Hep3B, MHCC-97H, MHCC-97L and Huh-7 cell lines, and the THLE2 cell line were obtained from the Cell Bank of Type Culture Collection of the Chinese Academy of Sciences. The HCC cell lines (Hep3B, MHCC-97H, MHCC-97L and Huh-7) were cultured in DMEM supplemented with $10 \%$ FBS, whereas the normal liver cell line (THLE2) was cultured in Bronchial Epithelial Cell Growth Medium supplemented with 10\% FBS (all from Gibco; Thermo Fisher Scientific, Inc.); all cells were maintained in a humidified incubator containing $5 \% \mathrm{CO}_{2}$ at $37^{\circ} \mathrm{C}$.

Hep3B and Huh-7 cell lines $\left(2 \times 10^{5}\right)$ were transfected with $50 \mathrm{nM}$ small interfering (si)RNA against CDC20 (si-CDC20) (Guangzhou RiboBio Co., Ltd.). The sequences for the siRNAs were as follows: si-CDC20 forward, 5'-GGAUUGGAGUUC UGGGAAUTT-3' and reverse, 5'-AUUCCCAGAACUCCA AUCCTT-3'; and siRNA-negative control (si-NC; scrambled siRNA) forward, 5'-UUCUUCGAACGUGUCACGUTT-3' and reverse, 5'-ACGUGACACGUUCGGAGAATT-3'. The cells were transiently transfected using Lipofectamine ${ }^{\circledR} 3000$ reagent (Invitrogen; Thermo Fisher Scientific, Inc) and the transfection was maintained for $>24 \mathrm{~h}$. Subsequent experiments were performed $72 \mathrm{~h}$ after transfection.

$R N A$ extraction and reverse transcription-quantitative $P C R$ $(R T$ - $q P C R)$. Briefly, total RNA was extracted from HCC tissues, adjacent tissues and cell lines using TRIzol ${ }^{\circledR}$ reagent (Invitrogen; Thermo Fisher Scientific, Inc.) according to the manufacturer's protocol. RT to cDNA was performed using a PrimeScript RT reagent (Takara Bio, Inc.), and qPCR was performed using SYBR Premix Ex Taq II (Takara Bio, Inc.) and a LightCycler system (Roche Diagnostics). The temperature protocol for RT was as follows: $25^{\circ} \mathrm{C}$ for $10 \mathrm{~min}$, followed by $45^{\circ} \mathrm{C}$ for $30 \mathrm{~min}$ and $80^{\circ} \mathrm{C}$ for $30 \mathrm{~min}$. The primer sequences were as follows: CDC20 forward, 5'-GACCACTCCTAG CAAACCTGG-3' and reverse, 5'-GGGCGTCTGGCTGTT TTCA-3'; $\beta$-actin forward, 5'-GGATGCAGAAGGAGATCA CTG-3' and reverse, 5'-CGATCCACACGGAGTACTTG-3'. The following thermocycling conditions were used for qPCR: Initial denaturation at $95^{\circ} \mathrm{C}$ for $10 \mathrm{~min}$, followed by 40 cycles at $90^{\circ} \mathrm{C}$ for $30 \mathrm{sec}$ and $60^{\circ} \mathrm{C}$ for $30 \mathrm{sec}$, and a final extension step at $72^{\circ} \mathrm{C}$ for $10 \mathrm{~min}$. $\beta$-actin was used as the internal 
Table I. Association between CDC20 expression and clinicopathological features of patients with hepatocellular carcinoma.

\begin{tabular}{|c|c|c|c|c|}
\hline \multirow[b]{2}{*}{ Variables } & \multirow[b]{2}{*}{ Cases } & \multicolumn{2}{|c|}{ CDC20 expression } & \multirow[b]{2}{*}{ P-value } \\
\hline & & Low $(n=29)$ & High $(n=42)$ & \\
\hline Age, years & & & & 0.801 \\
\hline$<50$ & 33 & 14 & 19 & \\
\hline$\geq 50$ & 38 & 15 & 23 & \\
\hline Sex & & & & 0.901 \\
\hline Male & 30 & 12 & 18 & \\
\hline Female & 41 & 17 & 24 & \\
\hline TNM stage & & & & 0.163 \\
\hline $\mathrm{I} / \mathrm{II}$ & 37 & 18 & 19 & \\
\hline III/IV & 34 & 11 & 23 & \\
\hline Tumor size, cm & & & & 0.003 \\
\hline$\leq 5$ & 29 & 18 & 11 & \\
\hline$>5$ & 42 & 11 & 31 & \\
\hline $\mathrm{HBsAg}$ & & & & 0.233 \\
\hline Positive & 45 & 16 & 29 & \\
\hline Negative & 26 & 13 & 13 & \\
\hline AFP, ng/ml (17) & & & & 0.629 \\
\hline$\leq 20$ & 27 & 12 & 15 & \\
\hline$>20$ & 44 & 17 & 27 & \\
\hline Liver cirrhosis & & & & 0.541 \\
\hline Present & 47 & 18 & 29 & \\
\hline Absent & 24 & 11 & 13 & \\
\hline Number of tumors & & & & 0.002 \\
\hline Single & 36 & 21 & 15 & \\
\hline Multiple ( $\geq 2$ ) & 35 & 8 & 27 & \\
\hline Vascular invasion & & & & 0.030 \\
\hline Present & 33 & 9 & 24 & \\
\hline Absent & 38 & 20 & 18 & \\
\hline
\end{tabular}

AFP, $\alpha$-fetoprotein; CDC20, cell division cycle 20; HBsAg, hepatitis B surface antigen.

reference gene, and the mRNA expression levels of CDC20 were analyzed using the $2^{-\Delta \Delta \mathrm{Cq}}$ method (18).

Western blotting. Total protein was extracted from cell lines $\left(1 \times 10^{6}\right)$ and tissues using a 100:1 mixed solution of RIPA buffer (Beyotime Institute of Biotechnology) and PMSF (Beyotime Institute of Biotechnology). The protein concentrations were quantified using the Bradford protein assay (Bio-Rad Laboratories, Inc.). Equal concentrations of protein (30 $\mu \mathrm{g} /$ lane) were separated by SDS-PAGE on a $10 \%$ gel and transferred to PVDF membranes. The membranes were then blocked with $5 \%$ nonfat dried milk at room temperature for $1 \mathrm{~h}$ and were probed at $4^{\circ} \mathrm{C}$ overnight with primary antibodies against CDC20 (1:2,000; cat. no. ab183479;), N-cadherin (1:5,000; cat. no. ab76011), vimentin (1:5,000; cat. no. ab92547), E-cadherin (1:100; cat. no. ab40772), Ki-67 (1:5,000; cat. no. ab92742) and $\beta$-actin (1:5,000; cat. no. ab8227) (all from Abcam). After washing, the membranes were incubated with the appropriate HRP-conjugated secondary antibodies (1:5,000; cat. no. ab6721; Abcam) at room temperature for $1 \mathrm{~h}$. Finally, immunoreactive protein bands were visualized using an enhanced chemiluminescence solution (EMD Millipore) and a ChemiDoc Imaging system (Bio-Rad Laboratories, Inc.). $\beta$-actin was used as the internal control. Protein expression was semi-quantified using Quantity One version 4.6 software (Bio-Rad Laboratories, Inc.).

Bioinformatics analysis. The Gene Expression Profiling Interactive Analysis (GEPIA) (19) database (http://gepia. cancer-pku.cn/index.html) was used to analyze the expression levels of CDC20 in unpaired non-tumor liver tissue samples and HCC tissue samples. The threshold settings were set to $\mathrm{P}<0.01$, fold change $\geq 1$ (19).

Cell immunofluorescence. Hep3B and Huh-7 cells were seeded $\left(1 \times 10^{5}\right.$ cells $)$ and cultured on coverslips. After si-CDC20 transfection, Hep3B and Huh-7 cells were fixed with $4 \%$ paraformaldehyde at room temperature for $30 \mathrm{~min}$, and permeabilized with $0.25 \%$ Triton X-100 solution at room temperature for $15 \mathrm{~min}$. Subsequently, Hep3B and Huh-7 cells were washed with PBS and then blocked with 5\% bovine serum albumin (OriGene Technologies, Inc.) at room temperature for $1 \mathrm{~h}$. The coverslips were incubated with anti-CDC20 (1:500; cat. no. ab215908; Abcam) overnight at $4^{\circ} \mathrm{C}$. After washing with PBS, cells were incubated with an appropriate goat anti-rabbit secondary antibody (1:100; cat. no. ZF-0311; OriGene Technologies, Inc.) at $37^{\circ} \mathrm{C}$ for $30 \mathrm{~min}$ and DAPI. The slides were imaged using an inverted fluorescence microscope and results were recorded.

Cell Counting Kit-8 (CCK-8) assay. Hep3B and Huh-7 cells ( $2 \times 10^{3}$ cells/well) were transfected with si-CDC20 or si-NC for $48 \mathrm{~h}$ and cultured in a 96-well plate for 24, 48 and $72 \mathrm{~h}$. The CCK-8 assay (Dojindo Molecular Technologies, Inc.) was used to detect cell proliferation according to the manufacturer's protocol.

5-Ethynyl-2'-deoxyuridine (EdU) assay. Hep3B and Huh-7 cells $\left(1 \times 10^{3} /\right.$ well) were transfected with si-CDC20 or si-NC for $48 \mathrm{~h}$ and cultured in a 96-well plate. Hep3B and Huh-7 cells were incubated with $50 \mu \mathrm{M}$ EdU, $100 \mu \mathrm{l} 1 \mathrm{X}$ ApolloR reaction cocktail (cat. no. 100T; Guangzhou RiboBio Co., Ltd.) and $100 \mu \mathrm{l} 1 \mathrm{X}$ Hoechst 33342 for $30 \mathrm{~min}$ at $37^{\circ} \mathrm{C}$. Hep3B and Huh-7 cell proliferation was analyzed by counting the mean number of cells in three fields for each sample using a fluorescence microscope (magnification, x100).

Cell migration and invasion assays. After si-CDC20 transfection, Hep3B and Huh-7 cells were resuspended in high-glucose DMEM containing $1 \%$ FBS and seeded into the upper Transwell chamber at a density of $1 \times 10^{5}$ cells/well. Transwell chambers (Corning, Inc.) were used to detect cell invasion and migration. For migration assays, $500 \mu \mathrm{l}$ high-glucose DMEM containing $10 \% \mathrm{FBS}$ was added to the lower chamber. After incubation for $12 \mathrm{~h}$ at $37^{\circ} \mathrm{C}$, the Transwell chambers were fixed with $4 \%$ methanol at room temperature for $30 \mathrm{~min}$ and stained with $0.1 \%$ crystal violet for $20 \mathrm{~min}$ at room temperature. For 


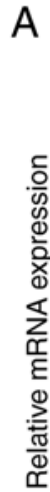

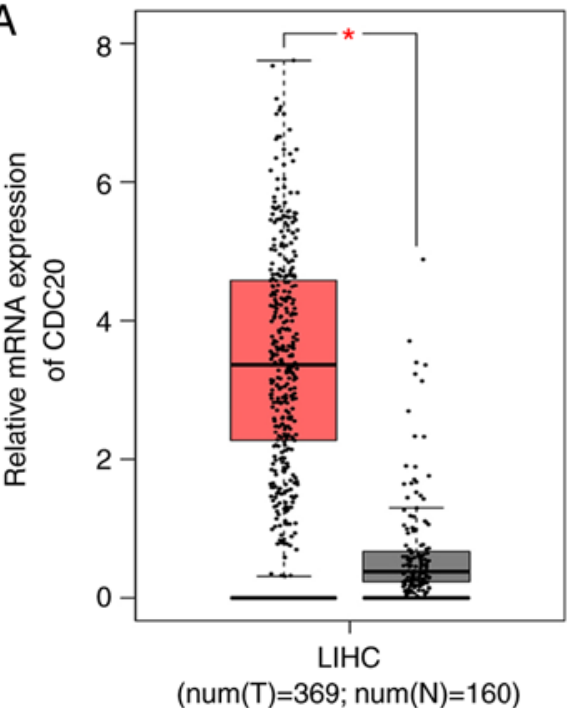

C

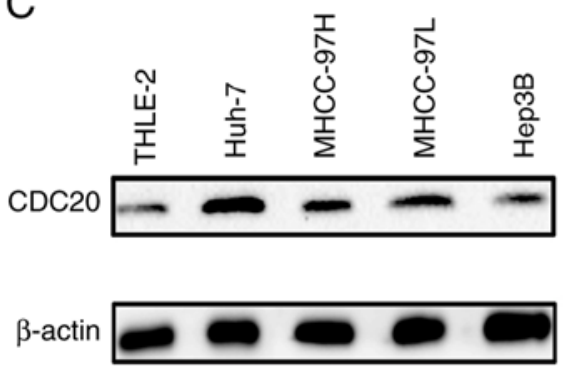

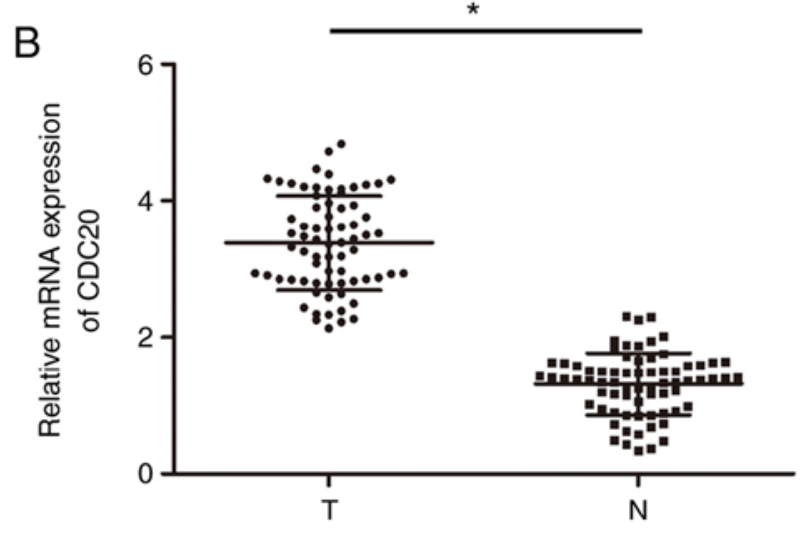

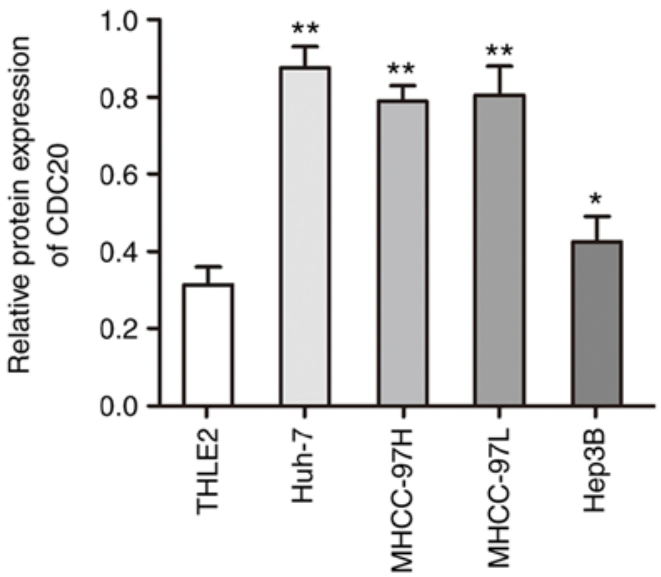

Figure 1. CDC20 expression in HCC tissues and HCC cell lines. (A) mRNA expression levels of CDC20 in HCC and adjacent noncancerous tissues were analyzed by Gene Expression Profiling Interactive Analysis. (B) mRNA expression levels of CDC20 in HCC and adjacent noncancerous tissues were analyzed in 71 clinical HCC samples. (C) Protein expression levels of CDC20 in HCC cells. ${ }^{*} \mathrm{P}<0.05,{ }^{* *} \mathrm{P}<0.01$ vs. THLE2 or as indicated. CDC20, cell division cycle 20 ; HCC, hepatocellular carcinoma; N, normal tissue; T, tumor tissue; LIHC, liver hepatocellular carcinoma.

the invasion assay, the inserts were precoated with Matrigel $(1 \mathrm{mg} / \mathrm{ml})$. After incubation for $16 \mathrm{~h}$ at $37^{\circ} \mathrm{C}$, the Transwell chambers were fixed with $4 \%$ paraformaldehyde at room temperature for $30 \mathrm{~min}$ and stained with $0.5 \%$ crystal violet at room temperature for $20 \mathrm{~min}$. Finally, invasive and migratory cells were counted under an inverted optical microscope (Olympus DP70 light microscope; Olympus Corporation; magnification, $\mathrm{x} 200)$.

Statistical analysis. All experiments were performed in triplicate. Statistical analyses were performed using SPSS version 22.0 software (IBM Corp.) and GraphPad Prism version 8.0 software (GraphPad Software, Inc.). The significant difference in CDC20 expression between HCC tissues and adjacent normal tissues was assessed using paired t-test. The relationship between CDC20 expression and clinicopathological characteristics was analyzed by $\chi^{2}$ test. Overall survival analysis was performed using the Kaplan-Meier method and the log-rank test. Univariate and multivariate Cox regression analyses were used to analyze the prognostic significance of CDC20. Statistical differences among multiple groups were analyzed by one-way ANOVA, followed by Tukey's test.
$\mathrm{P}<0.05$ was considered to indicate a statistically significant difference.

\section{Results}

CDC20 is highly expressed in HCC. The present study analyzed the expression levels of CDC20 in HCC using the GEPIA database; the results demonstrated that the mRNA expression levels of CDC20 were significantly higher in HCC tissues compared with those in paired adjacent tissues $(\mathrm{P}<0.05$; Fig. 1A). This finding was confirmed in collected HCC samples; the mRNA expression levels of CDC20 were higher in the HCC samples compared with those in the paired adjacent tissues $(\mathrm{P}<0.05$; Fig. 1B). Furthermore, the expression levels of CDC20 were higher in HCC cell lines compared with those in a normal liver cell line (THLE2) $(\mathrm{P}<0.05$; Fig. 1C).

CDC20 is associated with clinicopathological features of patients with HCC. Immunohistochemistry was used to detect the protein expression levels of CDC20 in HCC; the results indicated that high CDC20 expression was observed in 59.2\% of HCC samples (41/71) (Fig. 2A). Furthermore, the association 
Table II. Univariate and multivariate analyses of risk factors for overall survival in patients with hepatocellular carcinoma.

\begin{tabular}{|c|c|c|c|c|c|}
\hline \multirow[b]{2}{*}{ Variables } & \multirow[b]{2}{*}{$\mathrm{n}$} & \multicolumn{2}{|c|}{ Univariate analysis } & \multicolumn{2}{|c|}{ Multivariate analysis } \\
\hline & & HR $(95 \% \mathrm{CI})$ & $\mathrm{P}$-value & HR (95\% CI) & P-value \\
\hline Age, years & & $0.769(0.423-1.304)$ & 0.534 & & \\
\hline$<50$ & 33 & & & & \\
\hline$\geq 50$ & 38 & & & & \\
\hline Sex & & $0.506(0.347-0.863)$ & 0.364 & & \\
\hline Male & 30 & & & & \\
\hline Female & 41 & & & & \\
\hline TNM stage & & $1.338(0.450-4.338)$ & 0.673 & & \\
\hline $\mathrm{I} / \mathrm{II}$ & 37 & & & & \\
\hline III/IV & 34 & & & & \\
\hline Tumor size, $\mathrm{cm}$ & & $1.863(1.035-3.713)$ & 0.011 & $1.903(1.156-3.066)$ & 0.017 \\
\hline$\leq 5$ & 29 & & & & \\
\hline$>5$ & 42 & & & & \\
\hline HBsAg & & $1.304(0.408-4.368)$ & 0.364 & & \\
\hline Positive & 45 & & & & \\
\hline Negative & 26 & & & & \\
\hline AFP, ng/ml (17) & & $0.783(0.425-1.557)$ & 0.403 & & \\
\hline$\leq 20$ & 27 & & & & \\
\hline$>20$ & 44 & & & & \\
\hline Liver cirrhosis & & $0.933(0.489-1.806)$ & 0.869 & & \\
\hline Presence & 47 & & & & \\
\hline Absence & 24 & & & & \\
\hline Number of tumors & & $1.366(1.108-3.761)$ & 0.036 & $1.997(1.297-6.330)$ & 0.031 \\
\hline Single & 36 & & & & \\
\hline Multiple ( $\geq 2)$ & 35 & & & & \\
\hline Vascular invasion & & $0.673(0.339-1.208)$ & 0.574 & & \\
\hline Present & 33 & & & & \\
\hline Absent & 38 & & & & \\
\hline CDC20 expression & & $1.735(1.196-3.667)$ & 0.011 & $2.036(1.296-6.647)$ & 0.007 \\
\hline High & 29 & & & & \\
\hline Low & 42 & & & & \\
\hline
\end{tabular}

AFP, $\alpha$-fetoprotein; CDC20, cell division cycle 20; CI, confidence interval; HBsAg, hepatitis B surface antigen; HR, hazard ratio.

between CDC20 expression and the clinicopathological characteristics of patients with HCC was assessed. High CDC20 expression was revealed to be positively associated with tumor size $(\mathrm{P}=0.003)$, number of tumors $(\mathrm{P}=0.002)$ and vascular invasion $(\mathrm{P}=0.030)$ (Table $\mathrm{I})$.

CDC20 overexpression is associated with poor prognosis in $H C C$. The association between CDC20 expression and the poor prognosis of patients with $\mathrm{HCC}$ was analyzed using the 71 collected clinical HCC samples; the results revealed that high CDC20 expression was associated with significantly shorter overall patient survival compared with low CDC20 expression $(\mathrm{P}<0.01$; Fig. 2B). In addition, univariate analysis indicated that tumor size $(\mathrm{HR}=1.863 ; \mathrm{P}=0.011)$, multiplicity $(\mathrm{HR}=1.366 ; \mathrm{P}=0.036)$ and $\mathrm{CDC} 20$ expression $(\mathrm{HR}=1.735$; $\mathrm{P}=0.011)$ were significantly associated with overall survival in HCC (Table II). Multivariate analysis also suggested that tumor size $(\mathrm{HR}=1.903 ; \mathrm{P}=0.017)$, multiplicity $(\mathrm{HR}=1.997$; $\mathrm{P}=0.031)$ and $\mathrm{CDC} 20$ expression level $(\mathrm{HR}=2.036 ; \mathrm{P}=0.007)$ were independent prognostic factors for overall survival in HCC (Table II).

Knockdown of CDC2O inhibits the proliferation of Hep3B and Huh-7 cells. The present study used si-CDC20 to silence CDC20 expression. Hep3B and Huh-7 cells were used, as the expression levels of CDC20 were significantly different; the expression levels of CDC20 in Huh-7 were relatively high, whereas those in Hep3B cells were relatively low. Post-transfection with si-CDC20, the expression levels of CDC20 were significantly decreased in Hep3B and Huh-7 cells $(\mathrm{P}<0.05$; Fig. 3A and B). Furthermore, cellular immunofluorescence confirmed the reduction in the expression levels of 

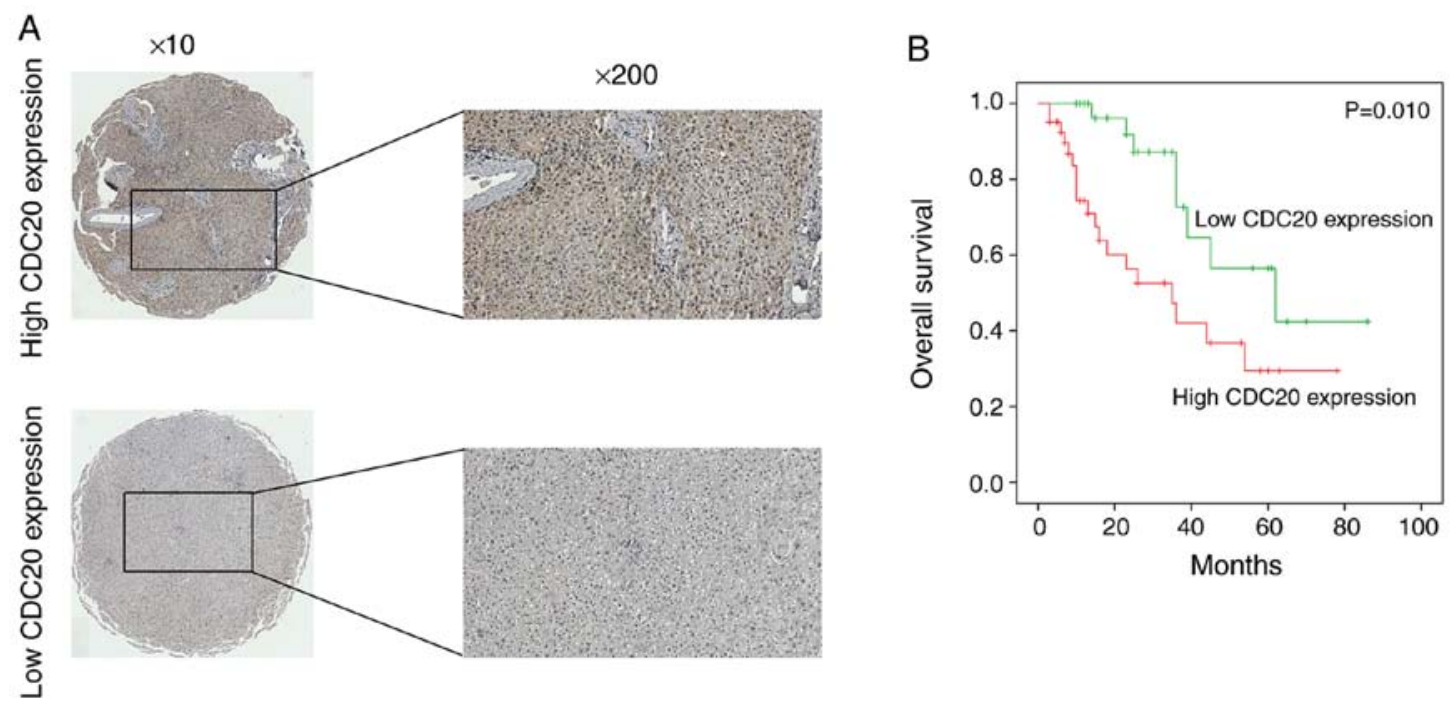

Figure 2. CDC20 expression and its relationship with prognosis in HCC. (A) Immunohistochemical staining analysis of CDC20 expression in HCC. (B) Kaplan-Meier analysis of overall survival of patients with HCC and high or low CDC20 expression (analyzed using the 71 clinical HCC samples collected). CDC20, cell division cycle 20; HCC, hepatocellular carcinoma.

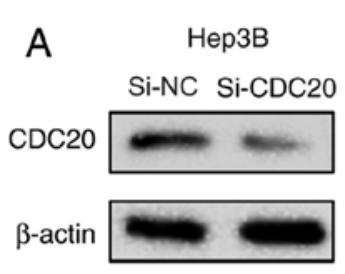

C
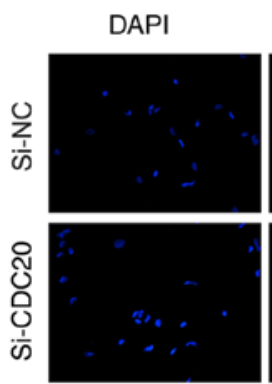
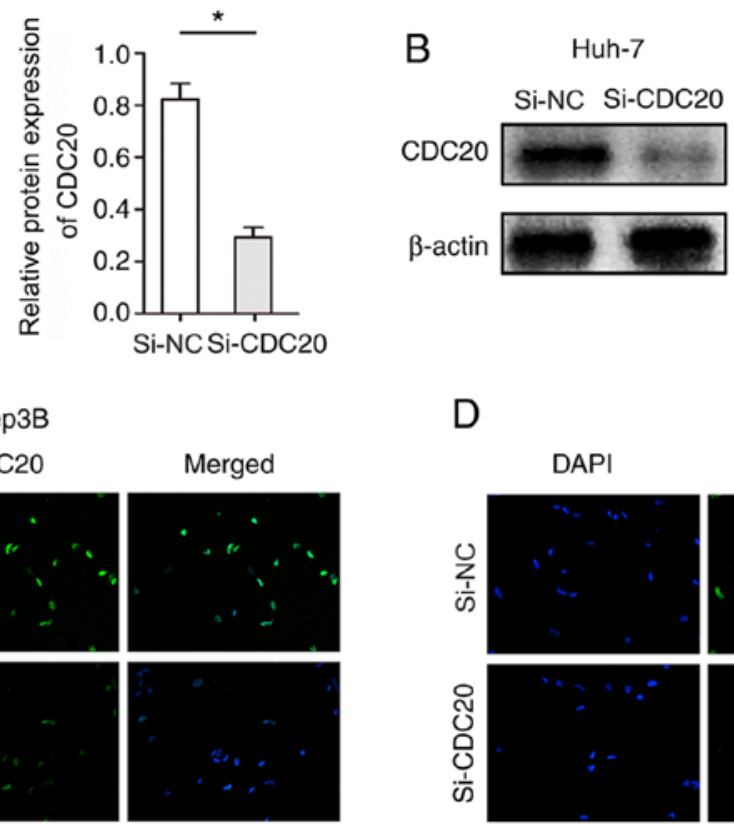

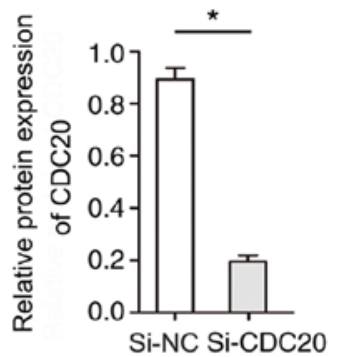

Huh-7
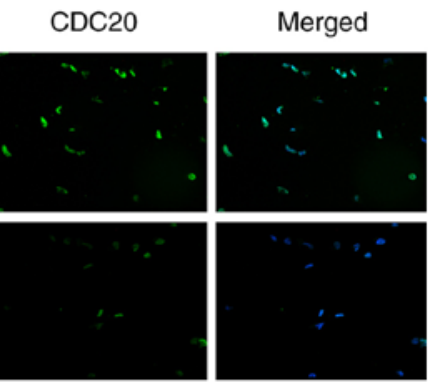

Figure 3. Knockdown of CDC20 in hepatocellular carcinoma cells. Protein expression levels of CDC20 in (A) Hep3B and (B) Huh-7 cells, as determined by western blotting. Protein expression of CDC20 in (C) Hep3B and (D) Huh-7 cells, as determined by immunofluorescence staining (magnification, x200). ${ }^{*} \mathrm{P}<0.05$. CDC20, cell division cycle 20 ; $\mathrm{NC}$, negative control; $\mathrm{Si}$, small interfering RNA.

CDC20 in Hep3B and Huh-7 cells transfected with si-CDC20 (Fig. 3C and D). The results of the CCK-8 assay revealed that knockdown of CDC20 significantly inhibited the proliferation of Hep3B and Huh-7 cells ( $\mathrm{P}<0.05$; Fig. $4 \mathrm{~A}$ and $\mathrm{B})$. In addition, the number of Hep3B and Huh-7 EdU-positive cells was significantly decreased in the si-CDC20 group compared with that in the si-NC group ( $\mathrm{P}<0.01$; Fig. $4 \mathrm{C}$ and $\mathrm{D})$.

Knockdown of CDC20 inhibits the migration and invasion of Hep3B and Huh-7 cells. The present study examined the effect of CDC20 on the migration and invasion of Hep3B and Huh-7 cells through Transwell assays. The results revealed that knockdown of CDC20 inhibited Hep3B and Huh-7 cell migration and invasion compared with those in the si-NC group $(\mathrm{P}<0.05$; Fig. 5A and $\mathrm{B})$.

Knockdown of CDC20 inhibits the expression of EMT-related proteins. The present study demonstrated that the proliferation, migration and invasion of HCC cells was reduced when expression of CDC20 was decreased. Therefore, whether the changes were related to alterations in EMT- and proliferation-associated proteins was assessed. The results of western blotting revealed that CDC20 silencing decreased $\mathrm{N}$-cadherin, vimentin and $\mathrm{Ki}-67$ expression levels, and increased E-cadherin expression levels compared with those in the si-NC group $(\mathrm{P}<0.05$; Fig. 6A and B). 

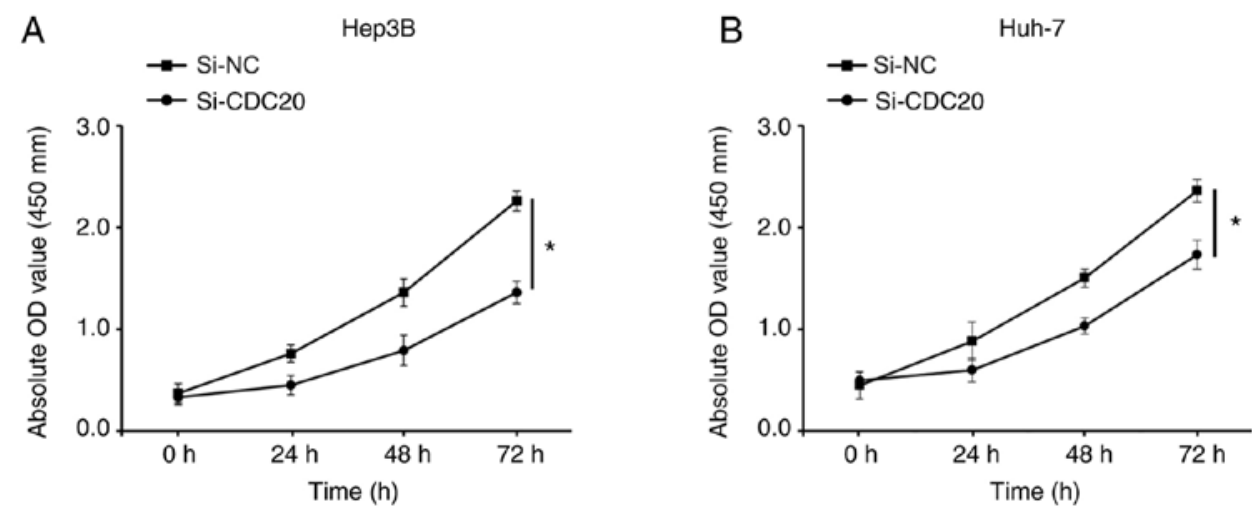

C

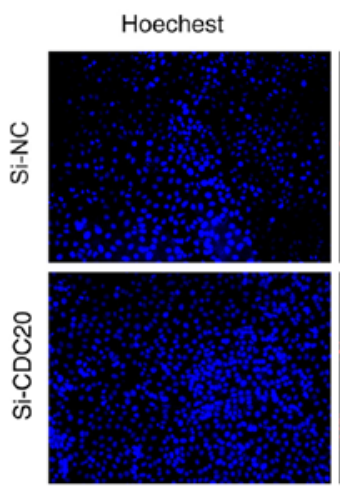

Нерзв
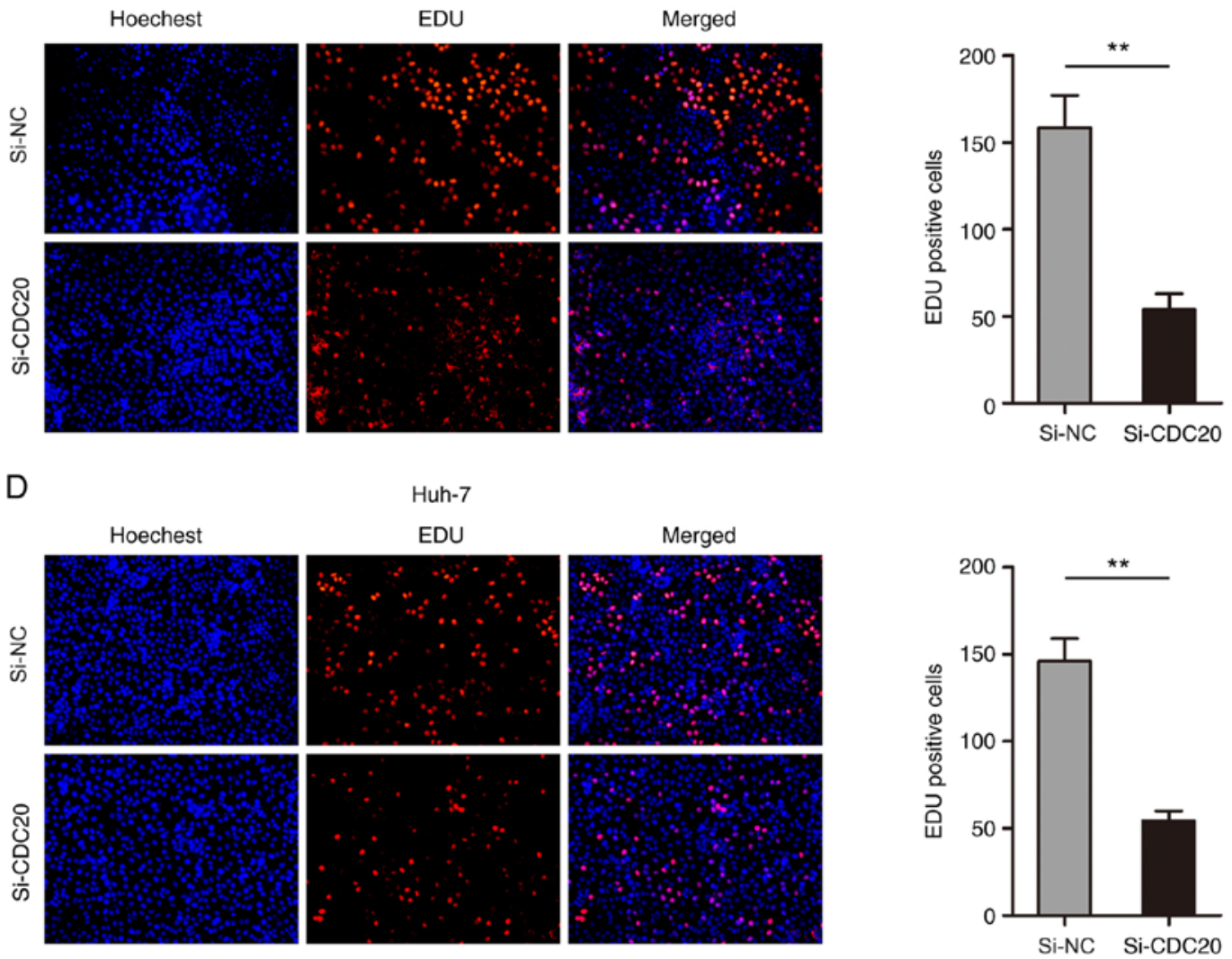

Figure 4. Effect of CDC20 on the proliferation of hepatocellular carcinoma cells. Cell Counting Kit- 8 assays were carried out to examine the effects of silencing CDC20 on the proliferative ability of (A) Hep3B and (B) Huh-7 cells. Cell proliferation was detected by EdU staining in (C) Hep3B and (D) Huh-7 cells after knockdown of CDC20 (magnification, $\mathrm{x} 200$ ). ${ }^{*} \mathrm{P}<0.05,{ }^{* * *} \mathrm{P}<0.01$. CDC20, cell division cycle 20; EdU, 5-ethynyl-2'-deoxyuridine; NC, negative control; $\mathrm{Si}$, small interfering RNA.

\section{Discussion}

The present study confirmed that CDC20 was highly expressed in HCC, and its high expression was significantly associated with the poor prognosis of patients with HCC. In addition, it was revealed that $\mathrm{CDC} 20$ could regulate the malignant biological behavior of HCC through EMT, which lays the foundation for further research into the specific mechanism underlying the effects of CDC20 on HCC.

CDC20 is a regulator of the APC, which can accelerate mitotic exit and interact with the spindle assembly checkpoint (SAC) (20). The SAC protein aggregates at the centromere in the prometaphase stage of mitosis and induces conformational changes of Mad2, which helps it bind to CDC20 and BubRl to form the mitotic checkpoint complex, an APC inhibitor (20). The high expression of CDC20 in tumor tissues has been reported to promote the malignant progression of tumors (21). Conversely, inhibition of CDC20 activity may regulate cell division cycle and accelerate cell apoptosis (21). A previous study demonstrated that decreasing the expression of CDC20 inhibited the migration of pancreatic cancer cells and metastatic breast cancer cells, and triterpene mixture extracted from the mushroom Poria cocos, purified triterpene dehydropropionic acid and polyvalerate $\mathrm{C}$ could significantly inhibit the expression of CDC20 to regulate the malignant biological behavior of tumor cells (22). In estrogen receptor-positive breast cancer, CDC20 mRNA was reported to be highly expressed, and high CDC20 expression was closely related to tumor size and poor tumor 
A

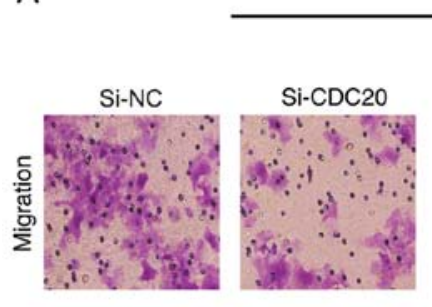

B

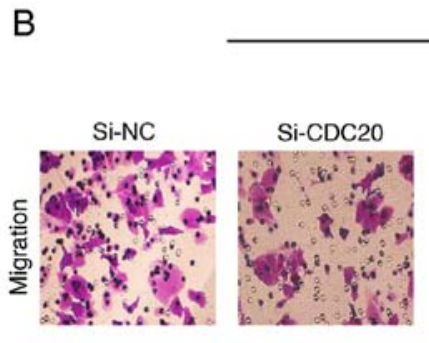

Нер3В

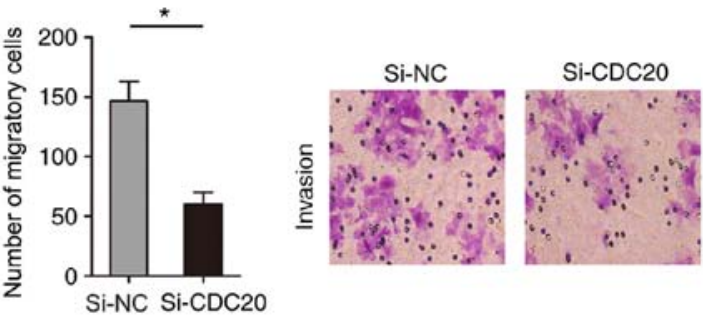

Huh-7

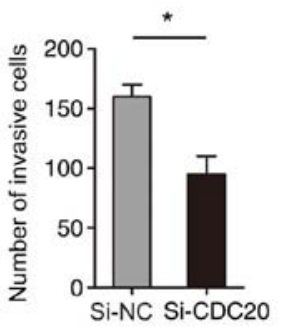

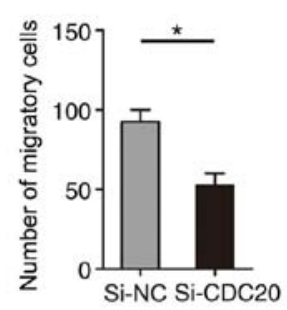

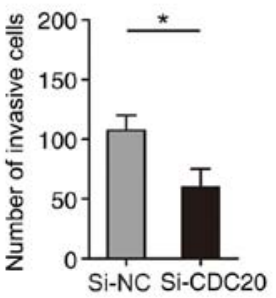

Figure 5. Effect of CDC20 on the migration and invasion of hepatocellular carcinoma cells. Migration and invasion assays were used to examine the effects of silencing CDC20 on (A) Hep3B and (B) Huh-7 cell migration and invasion (magnification, $\mathrm{x} 100$ ). ${ }^{*} \mathrm{P}<0.05$. CDC20, cell division cycle 20; NC, negative control; $\mathrm{Si}$, small interfering RNA.

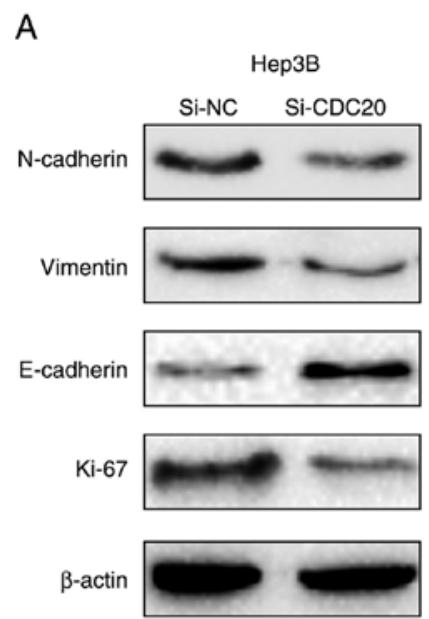

B

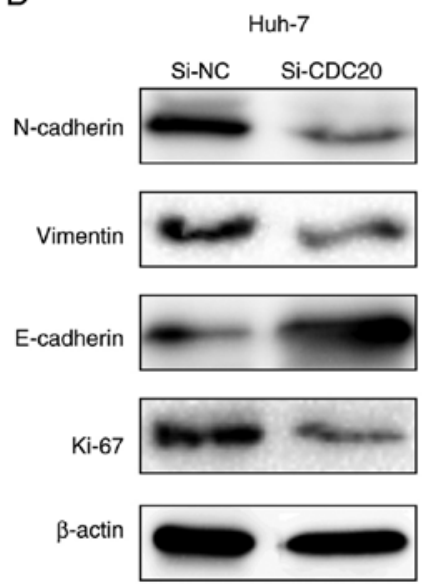

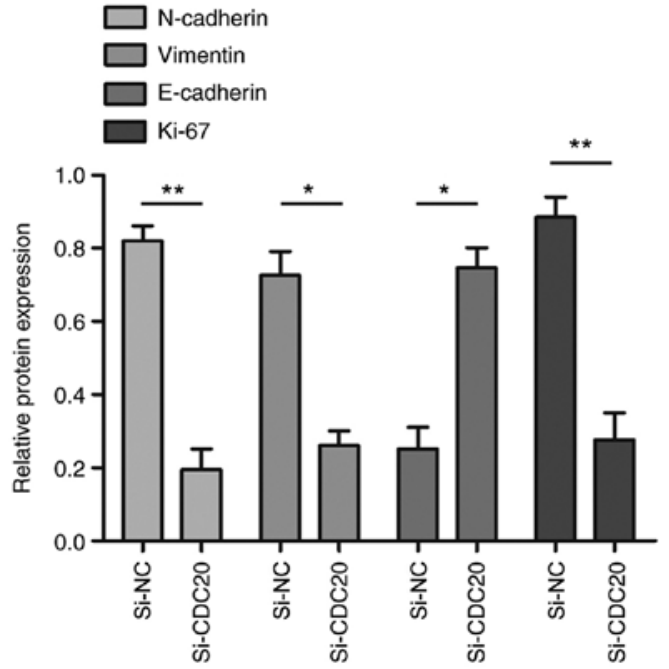

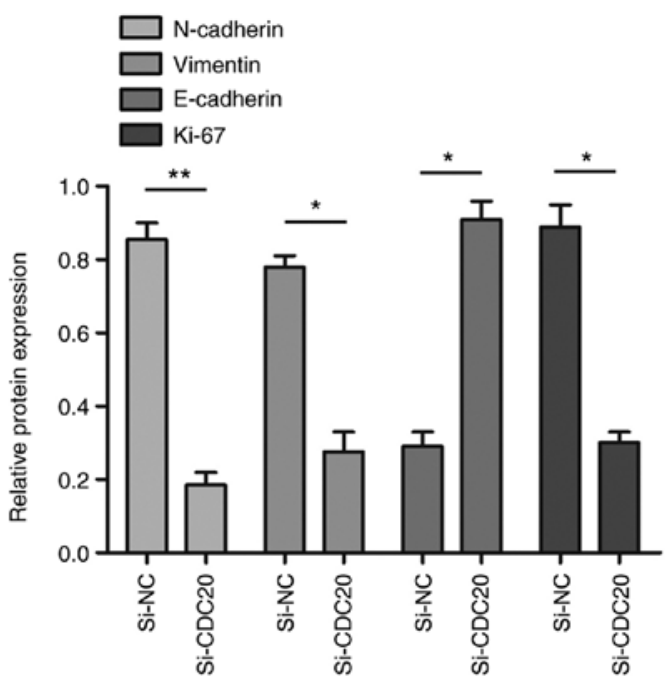

Figure 6. Effect of CDC20 on epithelial-mesenchymal transition in hepatocellular carcinoma cells. Protein expression levels of N-cadherin, vimentin, E-cadherin and Ki-67 after transfection of (A) Hep3B and (B) Huh-7 cells with si-CDC20. ${ }^{*} \mathrm{P}<0.05,{ }^{* *} \mathrm{P}<0.01$. CDC20, cell division cycle 20; NC, negative control; Si, small interfering RNA. 
grade (23). High mRNA expression levels of CDC20 were also significantly associated with poor prognosis. Notably, the high expression of CDC20 has been reported to be closely associated with the adverse effects of endocrine therapy in patients receiving hormone therapy; $\mathrm{CDC} 20$ is therefore considered an independent predictor of adverse clinical outcomes after endocrine therapy (23). A previous study also demonstrated that CDC20 expression was high and the expression of BIM was decreased in glioma cell lines resistant to temozolomide (24). Furthermore, inhibition of CDC20 expression could inhibit the EMT characteristics of drug-resistant cell lines and regulate the malignant growth of glioma drug-resistant cell lines (24). CDC20 has also been revealed to be highly expressed in primary cutaneous squamous cell carcinoma and may promote the malignant biological behavior of tumor cells through the Wnt/ $\beta$-catenin signaling pathway (25). In addition, CDC20 is a known key downstream gene of the MDM2-p53 signaling pathway in diffuse large B-cell lymphoma, and has been shown to regulate tumor cell proliferation, apoptosis and cell cycle changes (26). A previous study reported that CDC20 was very lowly expressed in normal liver tissues, and to the best of our knowledge, there are no reports of CDC20 expression in other liver diseases (1). Notably, the results of the present study demonstrated that CDC20 was highly expressed in HCC, and its high expression was significantly associated with the poor prognosis of patients with HCC. Our future studies aim to collect information on the expression of CDC20 in HCC from a public database, and further analyze the relationship between the expression of $\mathrm{CDC} 20$ and the poor prognosis of HCC.

One of the important biological characteristics of HCC is its high levels of metastasis, which can lead to poor prognosis of patients (27). At present, although the mechanism of tumor metastasis is not fully understood, existing studies have revealed that tumor metastasis is a complex process involving multiple factors and stages, which depends on the interaction between tumor cells and internal environmental factors, such as promoting tumor cell growth, invasion, migration and angiogenesis (28). A large number of studies have demonstrated that EMT may have an important role in tumor metastasis. EMT is a process during which epithelial cells lose polarity, tight junctions and adhesion junctions under the action of certain factors, and thus gain infiltrative and migratory abilities, and acquire interstitial cell morphology and characteristics (29-31). The loss of an epithelial phenotype and the acquisition of stromal characteristics are the main features of EMT. EMT includes the following: i) Decreased expression of cell adhesion molecules, which leads to the loss of epithelial cell intercellular adhesion, and ii) the keratin cytoskeleton is transformed into a vimentin cytoskeleton, and cells transform into spindle cells. In addition, EMT sometimes affects cell function and morphology $(30,31)$. This phenotypic transformation enables tumor cells to eliminate intercellular adhesion and thus become more aggressive.

The EMT process has been reported to be associated with changes in the expression levels of slug, snail and twist, which can regulate the malignant proliferation of HCC (32). Previous studies have reported that inhibition of CDC20 expression in cisplatin-resistant osteosarcoma cells significantly reversed the EMT phenotype and altered the expression of EMT biomarkers $(24,33)$. In estrogen receptor-positive luminal A breast cancer cells, EMT changes have been shown to serve an important regulatory role in breast cancer migration, invasion and metastasis (34). In the present study, CDC20 silencing decreased the expression levels of $\mathrm{N}$-cadherin, vimentin and $\mathrm{Ki}-67$, and increased E-cadherin expression suggesting that $\mathrm{CDC} 20$ expression may promote the metastasis of HCC through EMT. However, how this mechanism is achieved and the further regulatory mechanism require in-depth research. The present study demonstrated that $\mathrm{CDC} 20$ could regulate the malignant biological behavior of HCC through EMT, and these findings may provide a scientific and objective basis for further exploring the role of CDC20 in HCC from the perspective of EMT. Although changes in the expression level of CDC20 may cause changes in EMT, it is still unclear which specific molecule it acts on, and its specific regulatory mechanism is unclear. In addition, our future studies aim to analyze the mechanism of CDC20 in other liver diseases.

Research on CDC20 in HCC has mainly been conducted through bioinformatics analysis, and studies exploring the specific mechanism by which CDC20 affects HCC via cell function analysis are rare. The present study explored the role of CDC20 in HCC from the perspective of cell function and aimed to determine the specific molecular mechanism of CDC20. In addition, the relationship between CDC20 and the characteristics of patients with HCC was assessed. The present research is innovative and comprehensively analyzed the role of CDC20 in HCC. In conclusion, the present results indicated that the expression of CDC20 was significantly increased in HCC cells, and CDC20 overexpression was associated with poor HCC prognosis. Furthermore, CDC20 may promote proliferation, migration and invasion of HCC cells and could affect the biological function of HCC cells through EMT.

\section{Acknowledgements}

Not applicable.

\section{Funding}

The present study was funded by the Project of Department of Education, Sichuan Provincial (grant no. 16TDD00025), the Scientific Research Project of Affiliated Hospital of North Sichuan Medical College (grant no. 2020ZD001), the Scientific Research Project of Affiliated Hospital of North Sichuan Medical College (grant no. 2020JC035), Pre-research of State-level Project of North Sichuan Medical College (grant no. CBY19-YZ17) and the Popularization and Application Project of Sichuan Health Commission (grant no. 20PJ149).

\section{Availability of data and materials}

The datasets used and/or analyzed during the current study are available from the corresponding author on reasonable request.

\section{Authors' contributions}

GY, GW, YX and JL conceived and designed the experiments. GY, GW, YX and JS conducted experiments. GY, WL, TT and $\mathrm{JL}$ performed data analysis and wrote the paper. GY, GW, YX and JL confirm the authenticity of all the raw data. All authors read and approved the final manuscript. 


\section{Ethics approval and consent to participate}

All procedures involving human participants were approved by the Ethics Committee of Affiliated Hospital of North Sichuan Medical College, and complied with the 1964 Helsinki Declaration and its later amendments or comparable ethical standards. The participants signed an extensive informed consent form after being informed about the benefits and risks associated with the present study.

\section{Patients consent for publication}

Not applicable.

\section{Competing interests}

The authors declare that they have no competing interests.

\section{References}

1. Siegel RL, Miller KD and Jemal A: Cancer statistics, 2017. CA Cancer J Clin 67: 7-30, 2017.

2. Siegel RL, Miller KD and Jemal A: Cancer Statistics, 2019. CA Cancer J Clin 69: 7-34, 2019.

3. Llovet JM, Burroughs A and Bruix J: Hepatocellular carcinoma. Lancet 362: 1907-1917, 2004.

4. Jemal A, Bray F, Center MM, Ferlay J, Ward E and Forman D: Global cancer statistics. CA Cancer J Clin 61: 69-90, 2011.

5. Greten TF, Wang XW and Korangy F: Current concepts of immune based treatments for patients with HCC: From basic science to novel treatment approaches. Gut 64: 842-848, 2015.

6. Kapanidou M, Curtis NL and Bolanos-Garcia VM: Cdc20: At the Crossroads between chromosome segregation and mitotic exit. Trends Biochem Sci 42: 193-205, 2017.

7. Schrock MS, Stromberg BR, Scarberry L and Summers MK: APC/C ubiquitin ligase: Functions and mechanisms in tumorigenesis. Semin Cancer Biol 67: 80-91, 2020.

8. Dong S, Huang F, Zhang $\mathrm{H}$ and Chen Q: Overexpression of BUB1B, CCNA2, CDC20, and CDK1 in tumor tissues predicts poor survival in pancreatic ductal adenocarcinoma. Biosci Rep: Feb 26, 2019. doi: 10.1042/BSR20182306.

9. Parmar MB, K C RB, Löbenberg R and Uludağ H: Additive polyplexes to undertake siRNA therapy against CDC20 and Survivin in breast cancer cells. Biomacromolecules 19: 4193-4206, 2018.

10. De K, Grubb TM, Zalenski AA, Pfaff KE, Pal D, Majumder S, Summers MK and Venere M: Hyperphosphorylation of CDH1 in glioblastoma cancer stem cells attenuates $\mathrm{APC} / \mathrm{C}^{\mathrm{CDH} 1}$ activity and pharmacologic inhibition of APC/C ${ }^{\mathrm{CDH} 1 / \mathrm{CDC} 20}$ compromises viability. Mol Cancer Res 17: 1519-1530, 2019.

11. Kim Y, Choi JW, Lee JH and Kim YS: Spindle assembly checkpoint MAD2 and CDC20 overexpressions and cell-in-cell formation in gastric cancer and its precursor lesions. Hum Pathol 85: 174-183, 2019.

12. Li K, Mao Y, Lu L, Hu C, Wang D, Si-Tu J, Lu M, Peng S, Qiu J and Gao X: Silencing of CDC20 suppresses metastatic castration-resistant prostate cancer growth and enhances chemosensitivity to docetaxel. Int J Oncol 49: 1679-1685,2016.

13. Zhang Q, Huang H, Liu A, Li J, Liu C, Sun B, Chen L, Gao Y, $\mathrm{Xu}$ D and Su C: Cell division cycle 20 (CDC20) drives prostate cancer progression via stabilization of $\beta$-catenin in cancer stem-like cells. EBioMedicine 42: 397-440, 2019.

14. Giannelli G, Koudelkova P, Dituri F and Mikulits W: Role of epithelial to mesenchymal transition in hepatocellular carcinoma. J Hepatol 65: 798-808, 2016.
15. Diepenbruck M and Christofori G: Epithelial-mesenchymal transition (EMT) and metastasis: Yes, no, maybe? Curr Opin Cell Biol 43: 7-13, 2016.

16. Pastushenko I and Blanpain C: EMT transition states during tumor progression and metastasis. Trends Cell Biol 29: 212-226, 2019.

17. Wu H, Zhang W, Wu ZR, Liu Y, Shi Y, Gong J, Shen W and Liu C: MiR-29c-3p regulates DNMT3B and LATS1 methylation to inhibit tumor progression in hepatocellular carcinoma. Cell Death Dis 10: 48, 2019.

18. Livak KJ and Schmittgen TD: Analysis of relative gene expression data using real-time quantitative PCR and the 2(-Delta Delta C(T)) method. Methods 25: 402-408, 2001.

19. Tang Z, Li C, Kang B, Gao G, Li C and Zhang Z: GEPIA: A web server for cancer and normal gene expression profiling and interactive analyses. Nucleic Acids Res 45: W98-W102, 2017.

20. Zhou Z, He M, Shah AA and Wan Y: Insights into APC/C: From cellular function to diseases and therapeutics. Cell Div 11: 9, 2016.

21. McLean JR, Chaix D, Ohi MD and Gould KL: State of the APC/C: Organization, function, and structure. Crit Rev Biochem Mol Biol 46: 118-136, 2011.

22. Cheng S, Castillo V and Sliva D: CDC20 associated with cancer metastasis and novel mushroom-derived CDC20 inhibitors with antimetastatic activity. Int J Oncol 54: 2250-2256, 2019.

23. Alfarsi LH, Ansari RE, Craze ML, Toss MS, Masisi B, Ellis IO, Rakha EA and Green AR: CDC20 expression in oestrogen receptor positive breast cancer predicts poor prognosis and lack of response to endocrine therapy. Breast Cancer Res Treat 178: 535-544, 2019.

24. Wang J, Zhou F, Li Y, Li Q, Wu Z, Yu L, Yuan F, Liu J, Tian Y, Cao Y, et al: Cdc20 overexpression is involved in temozolomide-resistant glioma cells with epithelial-mesenchymal transition. Cell Cycle 16: 2355-2365, 2017.

25. Chu Z, Zhang X, Li Q, Hu G, Lian CG and Geng S: CDC20 contributes to the development of human cutaneous squamous cell carcinoma through the Wnt/ $\beta$-catenin signaling pathway. Int J Oncol 54: 1534-1544, 2019.

26. Sun C, Li M, Feng Y, Sun F, Zhang L, Xu Y, Lu S, Zhu J, Huang J, Wang J, et al: MDM2-P53 signaling pathway-mediated upregulation of $\mathrm{CDC} 20$ promotes progression of human diffuse large B-cell lymphoma. Onco Targets Ther 13: 10475-10487, 2020.

27. Couri T and Pillai A: Goals and targets for personalized therapy for HCC. Hepatol Int 13: 125-137, 2019.

28. Quail DF and Joyce JA: Microenvironmental regulation of tumor progression and metastasis. Nat Med 19: 1423-1437, 2013.

29. Yeung KT and Yang J: Epithelial-mesenchymal transition in tumor metastasis. Mol Oncol 11: 28-39, 2017.

30. Nieto MA, Huang RY, Jackson RA and Thiery JP: EMT: 2016. Cell 166: 21-45, 2016.

31. Chaffer CL, San Juan BP, Lim E and Weinberg RA: EMT, cell plasticity and metastasis. Cancer Metastasis Rev 35: 645-654, 2016.

32. Yu M, Xue H, Wang Y, Shen Q, Jiang Q, Zhang X, Li K, Jia M, Jia J, Xu J and Tian Y: MiR-345 inhibits tumor metastasis and EMT by targeting IRF1-mediated mTOR/STAT3/AKT pathway in hepatocellular carcinoma. Int J Oncol 50: 975-983, 2017.

33. Qiu E, Gao Y,Zhang B, Xia T,Zhang Z and Shang G: Upregulation of cell division cycle 20 in cisplatin resistance-induced epithelial-mesenchymal transition in osteosarcoma cells. Am J Transl Res 12: 1309-1318, 2020.

34. Xu Y, Qin L, Sun T, Wu H, He T, Yang Z, Mo Q, Liao L and $\mathrm{Xu} \mathrm{J}$ : Twist1 promotes breast cancer invasion and metastasis by silencing Foxa1 expression. Oncogene 36: 1157-1166, 2017.

This work is licensed under a Creative Commons Attribution-NonCommercial-NoDerivatives 4.0 International (CC BY-NC-ND 4.0) License. 